\title{
DENTABLE FUNCTIONS AND \\ RADIALLY UNIFORM QUASI-CONVEXITY
}

\author{
CARL G. LOONEY \\ Department of Mathematics \\ University of Toledo \\ Toledo, Ohio 43606 \\ U.S.A.
}

(Recieved April 18, 1978)

ABSTRACT. In this paper we give a further result which states sufficient conditions for the theory of convergence of minimizing sequences to be applicable, develop the theory further, and give an application.

KEY WORDS AND PHRASES. Denting points, dentable functions, radial quasiconvexity, normed spaces, convex domains, minimizing sequences.

AMS (MOS) SUBJECT CLASSIFICATION (1970) CODES. Primary 49045; Secondary 52A05, 46B99.

1. INTRODUCTION.

Let $X$ be a normed space, $C$ a closed bounded convex set in $X$, and $f: C \rightarrow R$

a (nonlinear) functional to be minimized on C. A minimizing sequence for $f$

on $C$ is a sequence $\left(x_{n}\right)$ in $C$ such that $f\left(x_{n}\right) \rightarrow \beta \equiv \inf f(C)$.

In [4] and [6], certain conditions were given which guarantee that any 
minimizing sequence of $\mathrm{f}$ on $\mathrm{C}$ will converge in norm to a minimum (when it exists). In this paper we give a further result which states sufficient conditions for the theory of [4] to be applicable, develop the theory further, and give an application.

\section{SOME DEFINITIONS AND THEOREMS}

The closed convex hull of a set $S$ in $X$ is denoted by $c 1-\operatorname{conv}(S)$. We say $S$ is dentable at $x \in S$ whenever: given any $\varepsilon>0, x \notin c 1-\operatorname{conv}\left(S \backslash B_{\varepsilon}(x)\right)$, where $\mathrm{B}_{\varepsilon}(\mathrm{x})$ is the open $\varepsilon$-ball centered on $\mathrm{x}$. In this case $\mathrm{x}$ is called a denting point of S [5] (strongly extremal point of S, [2; p. 97]). See [9] for the origin of the term "dentable".

A function $f$ on a convex set $C$ is said to be dentable [4] at $x_{0} \in C$ iff $\left(x_{0}, f\left(x_{0}\right)\right)$ is a denting point of epi $(f)=\{(x, \alpha) \in X \times R: \alpha \geqq f(x)\}$. It was shown in [4] that if $f$ is a 1.s.c. (lower semi-continuous) quasi-convex functional on a weakly compact convex set $C$ and has a unique minimum $x_{0} \epsilon \mathrm{C}$, then every minimizing sequence of $f$ converges in norm to $x_{0}$ iff $f$ is dentable at $x_{0}$. We say that $f$ is quasi-convex on $C$ iff the level sets $L_{\alpha}=\{x \in C$ : $f(x) \leqq \alpha\}$ are convex. This is equivalent to the following: for any $x, y \in C$, $f(\lambda x+(1-\lambda) y) \leqq \max \{f(x), f(y)\}, 0 \leqq \lambda<1$. Convex functionals are quasiconvex, but not conversely.

A normed space $X$, its closed unit ball, and its norm are all said to be $\underline{\text { uniformly }} \underline{\text { convex }}$ iff given $\varepsilon>0$ and $x, y$ with ||$x|| \leqq 1,\|y\| \leqq 1$ and || $\mathrm{x}-\mathrm{y} \|>\varepsilon$, there exists $\delta(\varepsilon)>0$ such that ||$\frac{1}{2} \mathrm{x}+\frac{1}{2} \mathrm{y}|| \leqq 1-\delta(\varepsilon)$. Every such

space is strictly convex, i.e., ||$\lambda \mathbf{x}+(1-\lambda) y \|<\lambda|| x||+(1-\lambda)|| y||$, $0<\lambda<1$. It is shown [3] that the $\ell^{\mathrm{p}}$ and $\mathrm{L}_{\mathrm{p}}$ spaces are uniformly convex for $1<\mathrm{p}<\infty$. When the modulus of convexity $\delta$ depends on the point $\mathrm{x}$ also, i.e., $\delta=(x, \varepsilon)>0$, then we say that $\|\cdot\|$ is 1ocally uniformly convex [8] . Locally uniformly convex spaces are not generally uniformly convex, but the converse is true. Also, locally uniformly convex spaces are strictly convex, 
but not conversely.

THEROEM 1. If $\mathrm{X}$ is a locally uniformly convex space, then every boundary point of $\bar{B}_{1}(0)$ is a denting point of $\bar{B}_{1}(0)$.

PROOF. Let $x$ have norm 1 . Given $\varepsilon>0$, let $Q_{\varepsilon}=\bar{B}_{1}(0)-B_{\varepsilon}(x)$. For any $\mathrm{y} \in \mathrm{Q}_{\varepsilon}, \quad|| \mathrm{y}-\mathrm{x} \|>\varepsilon$ so that there is some $\delta=\delta(\mathrm{x}, \varepsilon)>0$ such that ||$\frac{1}{2} \mathrm{x}+\frac{1}{2} \mathrm{y} \|$ $\leqq 1-\delta$. The set $\overline{\mathrm{B}}_{1-\delta}(0)$ can be strictly separated from $\mathrm{x}$ by a closed hyperplane (see, e.g., [3; p.193]) H which partitions $X$ into two halfspaces $H_{1}$ and $H_{2}$ with $\mathrm{H}_{1}$ being closed and containing $\mathrm{Q}_{\varepsilon} \cdot \mathrm{H}_{2}$ contains $\mathrm{x}$ as an interior point. Then c1-conv $\left(Q_{\varepsilon}\right) \subset{ }^{H}{ }_{1} \equiv$ closed convex set, so that $x \notin c 1-\operatorname{conv}\left(Q_{\varepsilon}\right)$.

LEMMA 1. Let $C$ be a compact convex subset and $f: C \rightarrow R$ be 1.s.c. and have a unique minimum $x_{0} \in C$. Then for any $\varepsilon>0$, $f$ is bounded away from $\beta=f\left(x_{0}\right)$ on $C-B_{\varepsilon}\left(x_{o}\right)$.

PROOF. The set $Q=C-B_{\varepsilon}\left(x_{0}\right)$ is compact and thus $f$ attains its inf fmum $\gamma$ on Q. By hypothesis, $\gamma>\beta$. Thus $f(x) \geqq \gamma>\beta$ on $Q$.

CONSTRUCTION. Let $\mathrm{f}$ assume its infimum $\beta$ at a unique point $\mathrm{x}_{0} \in \mathrm{C} \equiv$ closed bounded convex subset of a normed space $\mathrm{X}$ that is locally uniformly convex. The set $H=\{(x, \beta): x \in X\}$ meets epi(f) at the point $\left(x_{0}, \beta\right)$. Let $L$ be the vertical line $\left\{\left(x_{0}, \alpha\right): \alpha \in R\right\}$ in $X \times R$. Fix $r$ and take the point $\mathrm{p}_{r}=\left(x_{0}, \beta+r\right) \in L$ at a distance $r$ above $\left(x_{0}, \beta\right)$. Let $\bar{B}_{r}\left(P_{r}\right)$ be the closed ball of radius $r$ centered on $\mathrm{P}_{r}$. Then $\bar{B}_{r}\left(\mathrm{P}_{r}\right)$ meets $\mathrm{H}$ at the unique point $\left(\mathrm{x}_{0}, \beta\right)$.

Put $E_{r} \equiv\{(x, \alpha): \alpha \leq \beta+r\} \bigcap e p i(f)$.

THEOREM 2. Let $\mathrm{f}$ be a 1.s.c. functional on a convex bounded set $\mathrm{C}$ and let $f$ have a unique minimum $x_{0} \in C$. If $X$ is a locally uniformly convex normed space, then a sufficient condition for $f$ to be dentable at $x_{0}$ is that either,

i) there exist some $r>0$ such that $E_{r}$ be contained in $\bar{B}_{r}\left(P_{r}\right)$, or

ii) C be compact.

PROOF. i) Since $\left(x_{0}, f\left(x_{0}\right)\right)$ is a denting point of $B_{r}\left(P_{r}\right)$ in $x \times R$ (by 
Theorem 1 and the fact that the product norm ||$(x, \alpha)||=\left(|| x||_{x}^{2}+|\alpha|^{2}\right)^{\frac{1}{2}}$ remains locally uniformly convex) and ${ }_{r} \subset_{B_{r}}\left(p_{r}\right)$, it is clear that $\left(x_{0}, f\left(x_{0}\right)\right.$ ) $\notin c 1-\operatorname{conv}\left(E_{r}-B_{\varepsilon}\left(x_{0}, f\left(x_{0}\right)\right)\right) c c 1-\operatorname{conv}\left(B_{r}\left(p_{r}\right)-B_{\varepsilon}\left(x_{0}, f\left(x_{0}\right)\right)\right)$ for any $\varepsilon>0$.

ii) For any $\varepsilon>0$, $f$ is bounded away from $f\left(x_{0}\right)$ on $C-B_{\varepsilon}\left(x_{0}\right)$. Thus $f\left(x_{0}\right)$ is separated by a closed hyperplane from epi(f) $B_{\varepsilon}\left(x_{0}, f\left(x_{0}\right)\right)$.

The conditions of Theorem 2 part $i$ ) are strong, and as the next example shows, may not be satisfied even in a finite dimensional space. EXAMPLE 1. Let $f(x)=e^{-1 / x^{2}}$ for $x \neq 0$ and $f(0)=0$. Then $f$ is a continuous quasi-convex function $R$, but we take $C$ to be the compact set $[-1,1]$. The point $x=0$ is the unique minimum. Let $B_{r}\left(p_{r}\right)$ be centered on $p_{r}=(0, r)$ in $R^{2}$. The bottom branch of the sphere of $B_{r}\left(p_{r}\right)$ is a convex function $g(x)=$ $r-\sqrt{r^{2}-x^{2}}$. We show now that $\lim _{x \rightarrow 0} f(x) / g(x)=0$, so that $f(x)<g(x)$ on some nbhd of 0 . It can be shown that $f^{\prime}(0)=0=f^{\prime \prime}(0)$, by putting $t=1 / x$ and using the limit definition for derivatives at $x=0$. Then $\lim f(x) / g(x)=1$ im $f^{\prime \prime}(x) / g^{\prime \prime}(x)=0 /\left(r^{2}\right)^{-\frac{1}{2}}$ (by use of L'Hospital's Rule twice). Thus for any given $r$, there is some $\varepsilon>0$ such that epi(f) is not contained in $B_{r}\left(p_{r}\right)$ for $-\varepsilon<x<\varepsilon$. Although $f$ does not satisfy the hypothesis of Theorem 2, it is dentable by part ii of Theorem 2 .

We note that Theorem $2 i$ can be phrased in terms of the Gâteaux derivatives of $f$ and $g$, where $g(x)=\inf \left\{\alpha:(x, \alpha) \in \bar{B}_{r}\left(p_{r}\right)\right\}$, i.e., we need $\left|f^{\prime}(x ; y)\right| \geqq\left|g^{\prime}(x ; y)\right|$ at $x$ for all $y \in C$.

3. NEW TYPE OF QUASI-CONVEXITY AND AN IMPORTANT APPLICATION.

We say that a subset $S$ of a vector space is radially convex at $s_{0} \epsilon S$ iff any line $L$ through $s_{0}$ meets $S$ in a convex set $L \cap S$. Any convex set $C$ is radially convex at any point of $\mathrm{C}$.

A functional $f$ defined on $S$ is said to be, respectively, radially convex,

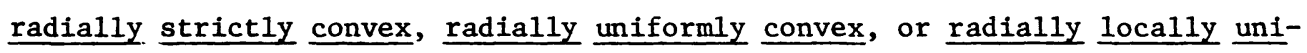


formly convex at $s_{0} \in \mathrm{S}$, whenever $\mathrm{S}$ is radially convex at $s_{0}$ and $\mathrm{f}$ is, resp., convex, strictly convex, uniformly convex, or locally uniformly convex on all segments $L \cap S$ through $s_{0}$. We replace "convex" by "quasi-convex" to get the resulting four new definitions for $f$ at $s_{0}$ in the radially convex set $\mathrm{S}$.

We now consider the space $C[\alpha, \beta]$ of all continuous functions on the interval $[\alpha, \beta]$ and the subset of rational functions. It is known(see [1] or [7]) that the approximation functional $T_{p}(a, b)=|| f(\cdot)-\left.r_{m n}(a, b ; \cdot)\right|_{p}$ is quasiconvex when $\mathrm{p}=\infty$, where $\mathrm{a} \in \mathrm{R}^{\mathrm{m}+1}, \mathrm{~b} \in \mathrm{R}^{\mathrm{n}+1}$, and $\mathrm{r}_{\mathrm{mn}}(\mathrm{a}, \mathrm{b} ; \mathrm{x})=\left(\mathrm{a}_{0}+\ldots+\right.$ $\left.a_{m} x^{m}\right) /\left(b_{0}+\ldots+b_{n} x^{n}\right)$. The norm $\|\cdot\|_{\infty}$ is not strictly convex since its graph on the unit ball contains horizontal line segments.

THEOREM 3. The functional $g \rightarrow|| g||$ is radially uniformly quasi-convex at 0 on any convex nbhd $U$ of $O$ in any normed space.

PROOF . Let $L$ be any line through 0 and put $L_{0}=L \cap U$. We know that $\|\cdot\|$ is convex on $L_{0}$. Now suppose that there are $x \neq y$ in $L_{0}$ such that ||$\frac{1}{2} x+\frac{1}{2} y \|=\max \{|| x||,|| y||\}$. Since $x, y \in I_{0}$, either $\||x| \mid<\| y \|(\|x\|$ $>\|\mathrm{y}\|$ is the same case) or else $\|x\|=\|y\|$. In this latter case $\frac{1}{2} x$ $+\frac{1}{2} y=0$, which yields a contradiction. Otherwise $\|x\|<\|y\|$ and $\|y\|=$ $\max \{|| x||,|| y||\}=\frac{1}{2}|| y||+\frac{1}{2}|| y||>\frac{1}{2}|| x||+\frac{1}{2}|| y|| \geq\left|\frac{1}{2} x+\frac{1}{2} y !\right|$, another contradiction. This completes the proof, since $\mathrm{L}_{0}$ is compact and strict quasiconvexity on $\mathrm{L}_{0}$ implies uniform quasi-convexity on $\mathrm{L}_{0}$ (see [4; Lemma 1]).

We note that a norm may not be radially strictly convex, e.g., $\|\cdot\| \|_{\infty}$ and $\|\cdot\|_{1}$ are linear on line segments from 0 to any $x$ ( $x$ must be in the positive orthant in the case of $\left.\|\cdot \mid\|_{1}\right)$. For $1<\mathrm{p}<\infty,\|\cdot\| \|_{\mathrm{p}}$ is uniformly convex and therefore is radially strictly convex on any radially convex set w.r.t. to a point.

THEOREM 4. The function $a \rightarrow\left\|f-\left(a_{0}+\ldots+a_{m} x^{m}\right)\right\|_{p}$ is radially uniformly quasi-convex at the minimum $\bar{a}=\left(\bar{a}_{0}, \ldots, \bar{a}_{m}\right)$ on any closed bounded nbhd of $\bar{a}$ for $\mathrm{f}$ fixed in $\mathrm{C}[\alpha, \beta], 1<\mathrm{p} \leqslant \infty$. 
PROOF. Without loss of generality, we assume that $f=0$. The linear function $a \rightarrow\left(a_{0}+\ldots+a_{m} x^{m}\right)$ followed by the convex map $\|\cdot\|$ is trivially convex with minimum on $R^{m+1}$ at 0 . Since the space of polynomials $P_{m}[\alpha, \beta]$ of degree $m$ or less is linearly isomorphic to $\mathrm{R}^{\mathrm{m}+1}$, a $\rightarrow\left\|a_{0}+\ldots+a_{m} x^{m}\right\|_{p}$ is actually a norm on $\mathrm{R}^{\mathrm{m}+1}$ and thus Theorem 2 holds (it is known that a unique minimum $\bar{a}$ does indeed exist for $1<\mathrm{p}$ ).

We now borrow a proposition from [7]. See [1] also.

PROPOSITION. For $f$ fixed in $C[\alpha, \beta]$, the functional $T_{\infty}(a, b)=\| f-r_{m n}$ $(a, b, \cdot)||_{\infty}$ is quasi-convex on any convex nbhd $U$ of the unique minimum ( $a *$, $\left.b^{*}\right)$, where $r_{m n}(a, b, x)=\left(a_{0}+\ldots+a_{m} x^{m}\right) /\left(b_{0}+\ldots+b_{n} x^{n}\right)$.

COROLLARY 1. $\mathrm{T}_{\infty}$ is radially uniformly quasi-convex at the minimum (a*, $\mathrm{b}^{*}$ ) of any closed nbhd $U$ in $\mathrm{R}^{\mathrm{m}+\mathrm{n}+2}$.

COROLLARY 2. Starting from any point $\left(a^{0}, b^{0}\right) \neq\left(a^{*}, b^{*}\right)$ there is a direction $d^{0}$ in which $T_{\infty}$ decreases. Further, given a step size of $\varepsilon^{0}$ there is a $\delta^{0}>0$ such that $\mathrm{T}_{\infty}$ will decrease by at least $\delta^{0}$ along $d^{0}$ until the directional minimum is reached.

COROLLARY 2. $\mathrm{T}_{\infty}$ is dentable at its unique minimum $\left(\mathrm{a}^{*}, \mathrm{~b}^{*}\right)$, and any minimizing sequence $\left(a^{n}, b^{n}\right)$ converges to $\left(a^{*}, b^{*}\right)$ 


\section{REFERENCES}

1. Barrodale, I. Best Rational Approximation and Strict Quasi-Convex1ty, SIAM J. Numerical Anal., 10 (1973) 8-12.

2. Choquet, G. Lectures on Analysis, Vo1. II, Benfamin, N.Y., 1969.

3. Köthe, G. Topological Vector Speces I, Springer-Verlag, Ber11n, 1969 (Trans1. of 1966 German Ed.).

4. Looney, C. G. Convergence of Minimizing Sequences, J. Math. Anal. \& App1.,61 (1977) 835-840.

5. Looney, C. G. A Krein-Milman Type Theorem for Certain Unbounded Convex Sets, J. Math. Analysis \& Appl., 48 (1974) 284-293.

6. Looney, C. G. Locally Uniformly Quas1-Convex Programaning, SIAM J. App1. Math. 28 (1975) 881-884.

7. Looney, C. G. Rational Approxination and Computation (preprint).

8. Lovaglia, A. R. Locally Uniformly Convex Banach Spaces, Trans. AMS (1965) 225-238.

9. Rieffel, M. A. Dentable subsets of Banach spaces with application to a Radon-Nikodym theorem, Proc. Conf. on Functional Analysis, U. C. Irvine, Thompson, Washington, D. C., 1967. 


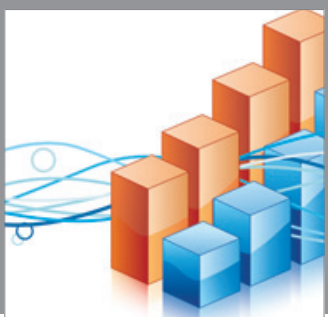

Advances in

Operations Research

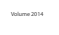

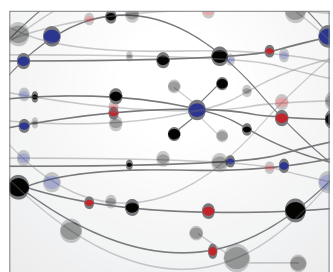

\section{The Scientific} World Journal
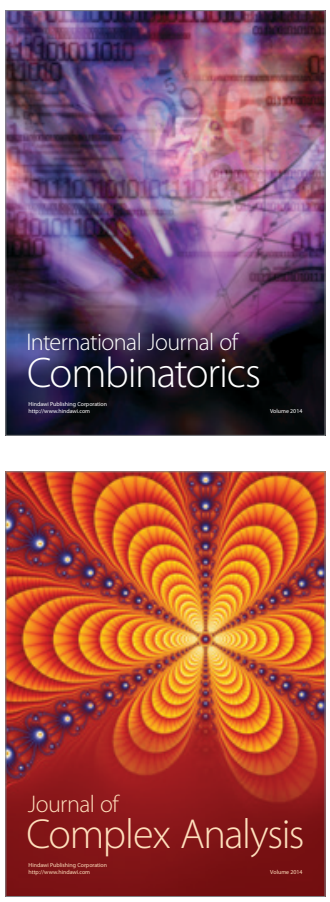

International Journal of

Mathematics and

Mathematical

Sciences
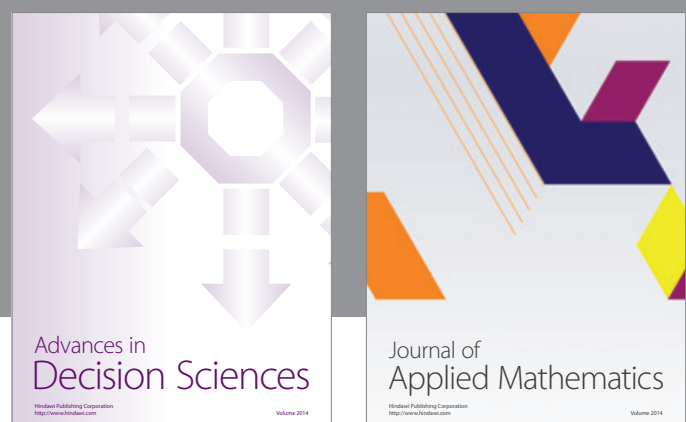

Journal of

Applied Mathematics
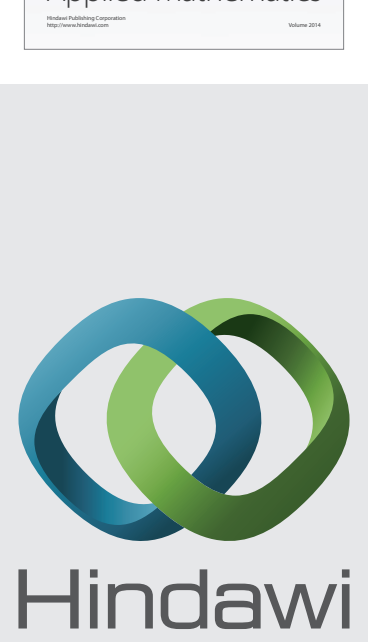

Submit your manuscripts at http://www.hindawi.com
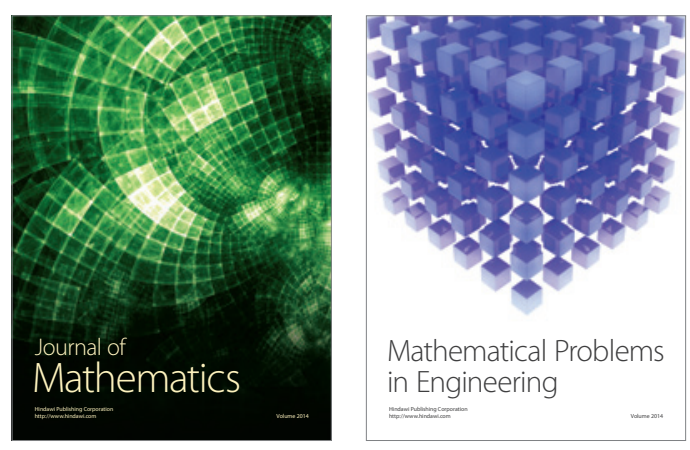

Mathematical Problems in Engineering
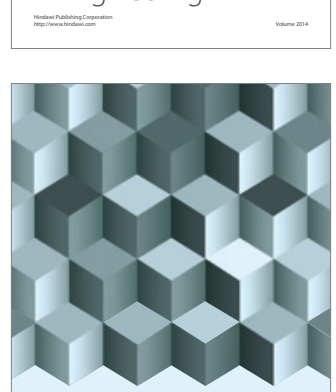

Journal of

Function Spaces
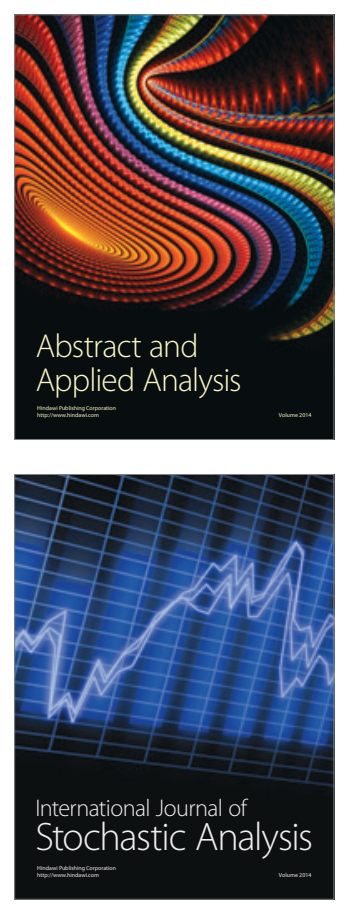

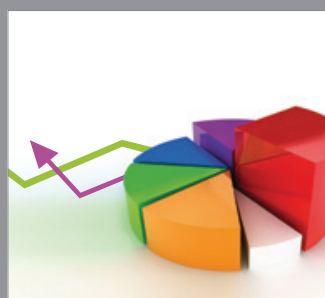

ournal of

Probability and Statistics

Promensencen
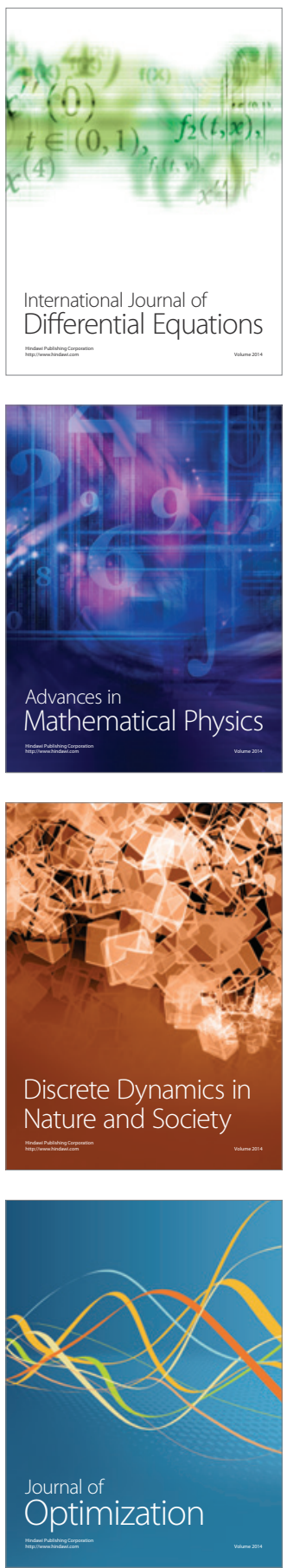